Research Article

\title{
Efficient Algorithms on Multicommodity Flow over Time Problems with Partial Lane Reversals
}

\author{
Urmila Pyakurel ${ }^{D},{ }^{1}$ Shiva Prakash Gupta, ${ }^{2}$ Durga Prasad Khanal, ${ }^{3}$ \\ and Tanka Nath Dhamala $\mathbb{D}^{1}$ \\ ${ }^{1}$ Central Department of Mathematics, Tribhuvan University, P.O. Box 13143, Kathmandu, Nepal \\ ${ }^{2}$ Tribhuvan University, Tri-Chandra Multiple Campus, Kathmandu, Nepal \\ ${ }^{3}$ Tribhuvan University, Saraswati Multiple Campus, Kathmandu, Nepal
}

Correspondence should be addressed to Tanka Nath Dhamala; amb.dhamala@daadindia.org

Received 14 July 2020; Accepted 1 October 2020; Published 31 October 2020

Academic Editor: Vladimir V. Mityushev

Copyright (c) 2020 Urmila Pyakurel et al. This is an open access article distributed under the Creative Commons Attribution License, which permits unrestricted use, distribution, and reproduction in any medium, provided the original work is properly cited.

\begin{abstract}
The multicommodity flow problem arises when several different commodities are transshipped from specific supply nodes to the corresponding demand nodes through the arcs of an underlying capacity network. The maximum flow over time problem concerns to maximize the sum of commodity flows in a given time horizon. It becomes the earliest arrival flow problem if it maximizes the flow at each time step. The earliest arrival transshipment problem is the one that satisfies specified supplies and demands. These flow over time problems are computationally hard. By reverting the orientation of lanes towards the demand nodes, the outbound lane capacities can be increased. We introduce a partial lane reversal approach in the class of multicommodity flow problems. Moreover, a polynomial-time algorithm for the maximum static flow problem and pseudopolynomial algorithms for the earliest arrival transshipment and maximum dynamic flow problems are presented. Also, an approximation solution to the latter problem is obtained in polynomial-time.
\end{abstract}

\section{Introduction}

The multicommodity flow problems concern with the routing of various commodities through a network to the specific source-sink pairs. For instance, message routing in telecommunications, railway and vehicle routine in transportation, and production planning and logistics support in general and in an emergency can be modeled as a multicommodity flow problem. For details and implementations, we refer to [1-4].

The transportation network is considered as a network associated with the transshipment of distinct commodities where the supply points (origins), the demand points (destinations), and the junction of road segments constitute the nodes. The connections between the two nodes signify the arcs. On each arc, the capacity that limits the flow amount (i.e., transported commodities) and the travel time are allocated.
The multicommodity flow problem can be classified into static and dynamic flow problems. The former one has been categorized as maximum, maximum concurrent, and minimum cost flow problems. The latter one has been categorized as maximum, maximum concurrent, quickest, and the earliest arrival multicommodity flow problems $[5,6]$.

If we do not distinguish the flow in the multicommodity flow problem, then it becomes a single-commodity flow problem. The dynamic flow (also known as flow over time) problem is introduced by Ford and Fulkerson [7]. In connection to this problem, Gale [8] proposed a more general problem known as the earliest arrival flow problem. The earliest arrival flow problem having multiple sources and sinks with given supplies and demands is the earliest arrival transshipment problem. Generally, a solution to this problem does not exist in the case of multiple sinks. 
However, it does always exists for multiple sources and a single-sink [9].

The multicommodity flow problem is more complex than their single-commodity part. Hall et al. [10] have shown that multicommodity flow over time is $\mathscr{N} \mathscr{P}$-hard even for series-parallel graphs or have only two commodities. Kappmeier [11] provided solutions of maximum multicommodity flow over time and multisource single-sink multicommodity earliest arrival transshipment problems using a time-expanded network within pseudopolynomialtime complexity.

Lane reversal implies flipping of arc orientations to amplify the flow and reduce the travel time by increasing its capacity. Flow models and strongly polynomial-time algorithms for two-terminal maximum and quickest flow problems were developed by Rebennack et al. [12]. The lane reversals are made at time zero and kept fixed afterward. The budget constraint problem with lane reversals is investigated in [13]. The optimal solutions of the earliest arrival flow in the two-terminal general network for both discrete and continuous-time settings can be found in [14-16]. Nath et al. [17] investigated the quickest contra-flowloc problem. By reversing the directions of arcs whenever necessary, a polynomial-time algorithm is presented by Pyakurel and Dhamala [18] for multisource single-sink earliest arrival transshipment. The major concern of partial lane reversals is to make use of the capacities of unused arcs in a network for other purposes. Pyakurel et al. [19] introduced the partial lane reversal strategy in which only essential arc capacities are used to extend the flow value. Dhamala et al. [20] investigated the quickest multicommodity flow problem with partial lane reversals. The saved capacities of unused arcs can be used for the logistic supports and facility location in emergency periods.

In this paper, we introduce the maximum static multicommodity flow (MSMCF), the maximum dynamic multicommodity flow (MDMCF), and the earliest arrival multicommodity transshipment (EAMCT) problems with partial lane reversals. We present efficient algorithms to solve these problems by reducing them into single-commodity flow problems and decomposing the flow along the paths.

The paper is organized as follows. In Section 2, we provide some basic notations and models used in the article. The MSMCF problem with partial lane reversals is introduced and an algorithm to solve the problem is presented in Section 3. The MDMCF problem with partial lane reversals is introduced in Section 4. In this section, we present algorithms to find a solution to this problem. The EAMCT problem and an algorithm to solve this problem is presented in Section 5. The paper is concluded in Section 6.

\section{Preliminaries}

The multicommodity flow problem consists of the shipping of distinct commodities from their respective origin nodes to corresponding destination nodes through a given network. Basic notations and mathematical formulations are given below.
2.1. Flow Models and Notations. Let a dynamic network topology be given by $\mathcal{N}=\left(V, A, K, u, \tau, d_{i}, S_{+}, S_{-}, T\right)$ with finite sets of nodes $V$, arcs $A$, and commodities $K=\{1,2, \ldots, k\}$, where $|V|=n$ and $|A|=m$. Each commodity $i \in K$ with demand $d_{i}$ is routed through a unique source-sink pair $\left(s_{i}, t_{i}\right)$. The sets $S_{+}$and $S_{-} \subset V$ denote source and sink sets of all commodities, respectively. On each arc $e=(v, w)$, the capacity function $u$ : $A \longrightarrow \mathscr{Z}_{\geq 0}$ restricts the flow of commodities and a nonnegative transit time function $\tau: A \longrightarrow \mathscr{Z}_{>0}$ measures the time to transship the flow from the entry point $v$ to the exit point $w$ of arc $e=(v, w)$. The time period $T$ is given in advance which is denoted by $\mathbb{}=$ $\{0,1, \ldots, T-1\}$ in discrete and $\mathbb{T}=[0, T)$ in continuoustime settings. A static network is a network besides the temporal dimension denoted by $\mathcal{N}=\left(V, A, K, u, d_{i}, S_{+}, S_{-}\right)$. Many nice properties developed primarily based on static network topology are building blocks for most of the realworld dynamic flow problems.

2.1.1. Static Multicommodity Flow. A multicommodity flow $\psi$ for the given static network $\mathcal{N}$ is the sum of all nonnegative static flows $\psi^{i}$ defined by the functions $\psi^{i}: A \longrightarrow \mathscr{R}^{+}$for each commodity $i$ satisfying

$$
\begin{aligned}
\operatorname{excess}_{\psi}^{i}(v) & = \begin{cases}-d_{i}, & \text { if } v=s_{i}, \\
0, & \text { if } v \notin\left\{s_{i}, t_{i}\right\}, \quad \forall i \in K, \\
d_{i}, & \text { if } v=t_{i},\end{cases} \\
0 & \leq \sum_{i \in K} \psi_{e}^{i} \leq u_{e}, \quad \forall e \in A,
\end{aligned}
$$

where

$$
\operatorname{excess}_{\psi}^{i}(v)=\sum_{e \in A^{\text {in }}(v)} \psi_{e}^{i}-\sum_{e \in A^{\text {out }}(v)} \psi_{e}^{i},
$$

represents the excess flow of commodity $i$ at node $v$. The sets $A^{\text {out }}(v)=\{(v, w) \mid w \in V\}$ and $A^{\text {in }}(v)=\{(w, v) \mid w \in V\}$ denote the outgoing arcs from node $v$ and the incoming arcs to node $v$, respectively, such that $A^{\text {out }}\left(S_{-}\right)=\varnothing$ and $A^{\text {in }}\left(S_{+}\right)=\varnothing$, except in the lane reversals. The second condition of the constraints in (1) are flow conservation constraints for each commodity at intermediate nodes. The constraints in (2) are bundle constraints that limit the flow of each commodity on the arc. The network flow is called circulation if it satisfies flow conservation at all the nodes, i.e., $\operatorname{excess}_{\psi}^{i}(v)=$ $0, \forall v \in V$. The maximum static multicommodity flow problem with $k$-commodities consist of $\psi_{1}, \psi_{2}, \ldots, \psi_{k}$ single-commodity flows maximizing $\sum_{i=1}^{k}\left|\psi^{i}\right|$ satisfying constraints (1) and (2).

A polynomial-time solution of the MSMCF problem has been obtained by linear programming techniques (e.g., ellipsoid method or interior-point method). In this problem, each commodity $i=1,2,3, \ldots, k$ has a unique source-sink pair $\left(s_{i}-t_{i}\right)$, and we wish to send the maximum flow from the source nodes $s_{i}$ to the sink nodes $t_{i}$ satisfying given bundle capacity $u_{e}$ on each $\operatorname{arc} e=(v, w)$. That is, we wish to maximize the sum of the flows over all the commodities. 
2.1.2. Dynamic Multicommodity Flow. For a given dynamic network $\mathcal{N}$ with constant transit time on arcs, a multicommodity flow over times $\xi$ is a sum of flows defined by $\xi^{i}: A \times \mathbb{T} \longrightarrow \mathscr{R}^{+}$satisfying constraints (4)-(6):

$$
\begin{aligned}
& \operatorname{excess}_{\xi}^{i}(v, T-1)= \begin{cases}-d_{i}, & \text { if } v=s_{i}, \\
0, & \text { if } v \notin\left\{s_{i}, t_{i}\right\}, \quad \forall i \in K, \\
d_{i}, & \text { if } v=t_{i},\end{cases} \\
& \operatorname{excess}_{\xi}^{i}(v, \theta) \geq 0, \quad \forall v \notin\left\{s_{i}, t_{i}\right\}, i \in K, \theta \in \mathbb{T}, \\
& 0 \leq \xi_{e}(\theta)=\sum_{i \in K} \xi_{e}^{i}(\theta) \leq u_{e}, \quad \forall e \in A, \theta \in \mathbb{T},
\end{aligned}
$$

where

$$
\operatorname{excess}_{\xi}^{i}(v, \theta)=\sum_{\delta=\tau_{e}}^{\theta} \sum_{e \in A^{\text {in }}(v)} \xi_{e}^{i}\left(\delta-\tau_{e}\right)-\sum_{\delta=0}^{\theta} \sum_{e \in A^{\text {out }}(v)} \xi_{e}^{i}(\delta),
$$

is the excess flow of commodity $i$ on node $v$ at time $\theta$. Here, the second condition of the constraints in (4) are flow conservation constraints at time horizon $T$, whereas nonconservation of flow at intermediate time points $\theta \in \mathbb{T}=$ $\{0,1, \ldots, T-1\}$ is represented by the constraints in (5). Similarly, the constraints in (6) are bundle constraints that limit the flow on each arc at each point of time. The aim is to maximize the amount of flow to satisfy the demand $d_{i}$ of each commodity $i$ from $s_{i}$ to $t_{i}$ which is stated in the first and last conditions of (4). The earliest arrival multicommodity flow problem is to find a dynamic multicommodity flow of maximum value $\operatorname{val}_{\text {max }}(\xi(\theta))$ for all time units $\theta \in\{0,1$, $\ldots, T-1\}$ defined by objective function:

$$
\operatorname{val} \xi(\theta)=\sum_{\delta=\tau_{e}}^{\theta} \sum_{e \in A_{t_{i}}^{\text {in }}} \xi_{e}^{i}\left(\delta-\tau_{e}\right)
$$

Instead of each time $\theta$, if the maximization is considered for a given time $\theta=T-1$, it is defined as an MDMCF problem.

The equality in (5) represents strong flow conservation constraints that do not allow to store the flow at intermediate nodes for each time $\theta$ (i.e., without intermediate storage). The strict inequality constraint in (5) represents the weak flow conservation constraint at intermediate nodes for all times with intermediate storage.

2.2. $\Delta$-Condensed Time-Expanded Graph. The network topology $\mathcal{N}=\left(V, A, K, u, \tau, d_{i}, S_{+}, S_{-}, T\right)$ is considered. Fleischer and Skutella [21] introduced the $\Delta$-condensed time-expanded network $\mathcal{N}_{T}^{\Delta}=\left(V_{T}^{\Delta}, A_{T}^{\Delta}=A_{M}^{\Delta} \cup A_{H}^{\Delta} \cup A_{+}^{\Delta} \cup\right.$ $\left.A_{-}^{\Delta}, K, u^{\prime}, \tau^{\prime}, d_{i}, S_{+}^{\prime}, S_{-}^{\prime}, T\right)$, where all transit times on arcs are multiples of $\Delta>0$ such that $\lceil T / \Delta\rceil$ is bounded by a polynomial in input size. The nodes and arcs in the $\Delta$-condensed time-expanded network are defined as

$$
\begin{aligned}
& V_{T}^{\Delta}=\left\{v_{\theta}: v \in V, \theta=0,1,2, \ldots,\lceil T / \Delta\rceil-1\right\} \cup\left\{s_{i}^{\prime}, t_{i}^{\prime}: i \in K\right\} \cup\left\{s^{*}, t^{*}\right\}, \\
& A_{M}^{\Delta}=\left\{\left(v_{\theta}, w_{\theta+\tau_{e}}\right): e=(v, w) \in A, \quad \theta=0,1, \ldots,\left\lceil\left(T-\tau_{e}\right) / \Delta\right\rceil-1\right\}, \\
& A_{H}^{\Delta}=\left\{\left(v_{\theta}, v_{\theta+1}\right): e=(v, w) \in A, \quad \theta=0,1, \ldots,\lceil T / \Delta\rceil-2\right\}, \\
& A_{+}^{\Delta}=\cup\left\{\left(s^{*}, s_{i}^{\prime}\right): i \in K\right\} \cup\left\{\left(s_{i}^{\prime}, s_{\theta}\right): i \in K, s_{i}^{\prime} \in S_{+}^{\prime}, \theta \in\{0,1,2, \ldots,\lceil T / \Delta\rceil-1\}\right\}, \\
& A_{-}^{\Delta}=\cup\left\{\left(t_{i}^{\prime}, t^{*}\right): i \in K\right\} \cup\left\{\left(t_{\theta}, t_{i}^{\prime}\right): i \in K, t_{i}^{\prime} \in S_{-}^{\prime}, \theta \in\{0,1,2, \ldots,\lceil T / \Delta\rceil-1\}\right\},
\end{aligned}
$$

where $S_{+}^{\prime}$ and $S_{-}^{\prime}$ are the super terminals for each commodity as well as for the $\Delta$-condensed time-expanded network, i.e., $\left\{s_{i}^{\prime}, t_{i}^{\prime}: i \in K\right\} \cup\left\{s^{*}, t^{*}\right\}$. The copies of $V_{T}^{\Delta}$ correspond to flow through $V$ in time $\mathbb{T}=\{\alpha \Delta\}$ for discrete-time and $\mathbb{T}=[\alpha \Delta$, $(\alpha+1) \Delta)$ for continuous-time, where $\alpha=\{0,1,2, \ldots$, $\lceil T / \Delta\rceil-1\}$. In this setting, for every arc corresponding to a discrete-time with a multiple of $\Delta$, capacities are rescaled by $\Delta u_{e}$. If arc transit times are not multiples of $\Delta$, then transit times are rounded up to a multiple of $\Delta$ by $\tau_{e}^{\prime}=\left\lceil\tau_{e} / \Delta\right\rceil \Delta$ and $0 \leq \tau_{e}^{\prime}-\tau_{e}<\Delta$ for all arcs $e \in A$. If we take $\Delta=1$, then the $\Delta$-condensed time-expanded network reduces to the classical time-expanded network.

2.3. Lane Reversal Technique. For a given network $\mathcal{N}$, the corresponding auxiliary network is denoted by $\mathscr{N}^{a}=(V$, $\left.A^{a}, K, u^{a}, \tau^{a}, d_{i}, S_{+}, S_{-}, T\right)$ with undirected edges in $A^{a}=\{(v, w):(v, w)$ or $(w, v) \in A\}$, where $e^{r}=(w, v)$ is the reversed arc of $e=(\nu, w)$. The capacity of the auxiliary arc is the sum of capacities of $\operatorname{arcs} e$ and $e^{r}$ such that $u_{e}^{a}=u_{e}+u_{e^{r}}$, where $u_{e}=0$ if $e \notin A$. The transit time of the auxiliary arc is

$$
\tau_{e}^{a}= \begin{cases}\tau_{e}, & \text { if } e \in A, \\ \tau_{e^{r}}, & \text { otherwise }\end{cases}
$$

Other network parameters are the same. The transformation of the multicommodity network with lane reversals is represented in Figures 1(a) and 1(b). The first, second, and third commodities are shipped through the paths $s_{1}-t_{1}$, $s_{2}-t_{2}$, and $s_{3}-t_{3}$, respectively.

Example 1. Let us consider a multicommodity network, where $s_{1}, s_{2}$, and $s_{3}$ are the source nodes and $t_{1}, t_{2}$, and $t_{3}$ are the sink nodes as shown in Figure 1(a). The arcs between nodes $v$ and $w$ denoted by $(v, w)$ and $(w, v)$ represent the two way of road segments. The first and second numbers on the arcs represent capacity and transit time (cost) associated 


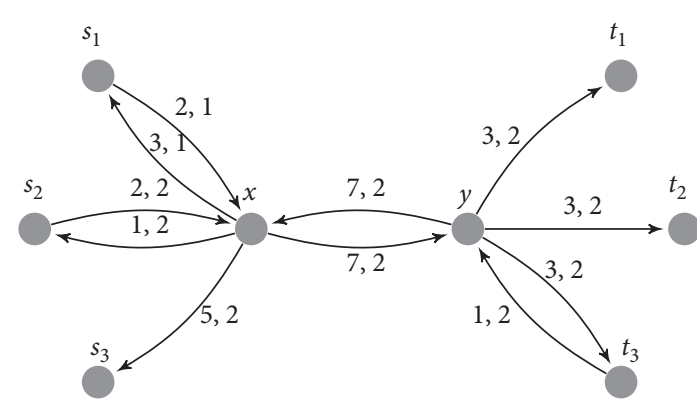

(a)



(b)

Figure 1: Auxiliary network (b) of the given network (a). (a) Capacity, transit time; (b) capacity, transit time.

with the arcs. By adding two-way capacities of the arcs $e$ and $e^{r}$ having the same transit time, an auxiliary network is formed with capacities $u^{a}$ and transit time $\tau^{a}$ as shown in Figure 1(b).

\section{Static Mutlicommodity Flow with Lane Reversals}

In this section, we introduce the partial lane reversals on the static multicommodity flow problem that makes best utilization of arc capacities to optimize the solution. The procedure for lane reversals is as follows:

(1) $\operatorname{Arc} e^{r}=(w, v)$ is reversed iff either $\psi_{e}-u_{e}>0$, where $e=(v, w)$, or there is $\psi_{e} \geq 0$ along the arc $e=(v, w) \notin A$. If $u_{e}^{a}-\psi_{e}>0$, then the $\operatorname{arc} e^{r}$ is reversed partially, and capacity of remaining $\operatorname{arcs} e^{r}$ is saved.

(2) If $\psi_{e}-u_{e}>0$ and $u_{e}^{a}-\psi_{e}=0$, then the $\operatorname{arc} e^{r}$ is reversed completely.

(3) If $\psi_{e}-u_{e}<0$ neither $e$ nor $e^{r}$ is reversed.

Problem 1. A network $\mathscr{N}=\left(V, A, K, u, S_{+}, S_{-}\right)$is considered. The MSMCF problem with partial lane reversals sends the maximum flow from the sources $s_{i}$ to the corresponding sinks $t_{i}$ in the unique pair $\left(s_{i}, t_{i}\right)$ for each commodity $i=$ $1,2, \ldots, k$ by saving the unused arc capacity.

\subsection{Polynomial-Time Solution of MSMCF Problem.} General linear programming approach (e.g., ellipsoid method or interior-point method) solves the static multicommodity flow problem in polynomial-time. To solve the MSMCF problem with partial lane reversals (problem 1), we present Algorithm 1.

Theorem 1. The MSMCF with partial lane reversal problem can be solved using Algorithm 1 optimally.

Proof. First, we show that Algorithm 1 is feasible. We can compute maximum multicommodity flow either by using the ellipsoid or interior-point methods. Therefore, Step 2 of the algorithm is feasible. Steps 1, 3, 4, 5, and 6 are feasible being transformation, decomposition, reversing arc, and saving arc capacities as in [19]. Hence, feasibility is shown.

Now, we prove the optimality of the algorithm. On the transformed network $\mathscr{N}^{a}$, the MSMCF is computed iteratively using an efficient algorithm. As flow is send in a unique pair $\left(s_{i}, t_{i}\right)$ for each commodity $i=1,2, \ldots, k$, the problem can be reduced to the static flow for a single commodity. In the static single-commodity flow, the maximum static flow on the transformed network is equivalent to the maximum static flow with partial lane reversals on the original network as in [19], and capacity of unused arcs is saved. Thus, the MSMCF in the transformed network is equal to the MSMCF with partial lane reversals for the original network.

Corollary 1. The MSMCF with partial lane reversals can be computed in polynomial-time complexity.

Proof. The complexity of the algorithm is dominated by Steps 2 and 3. All other steps can be computed in linear time. The maximum multicommodity flow problem is a linear programming problem, so the general linear programming technique (ellipsoid method or interior-point methods) solves the static multicommodity problem on the auxiliary network in polynomial-time in Step 2. This solution is equivalent to the maximum static multicommodity flow problem on the given network. The flow can be decomposed in $O(m n)$ time in Step 3. Hence, the MSMCF with partial lane reversals can be computed in polynomial-time.

Example 2. If we consider network as in Example 1 without temporal dimension, then it becomes a static multicommodity network. We can send the flow on an auxiliary network as shown in Figure 1(b). The maximum static multicommodity flow before lane reversals can be calculated in Figure 1(a) and after lane reversals in Figure 2(a). The saved capacity of unused arcs is shown in Figure 2(b).

The comparison of MSMCF before and after lane reversals is presented in Table 1.

Theorem 2. If a minimum cut in the multicommodity network has the symmetric capacity, then the flow value can be at most doubled with lane reversal reconfiguration. 
Input: given static multicommodity flow network $\mathcal{N}=\left(V, A, u, K, S_{+}, S_{-}\right)$

Output: the MSMCF with partial lane reversals

(1) The given network is transformed by adding two-way capacities as $\mathscr{N}^{a}=\left(V, A^{a}, u_{e}^{a}, K, S_{+}, S_{-}\right)$.

(2) Compute the MSMCF on the transformed network $\mathscr{N}^{a}$.

(3) Decompose the flow along the $s_{i}-t_{i}$ paths and cycles and remove flows in cycles $\forall i \in K$.

(4) Reverse $e^{r} \in A$ up to the capacity $\psi_{e}-u_{e}$ iff $\psi_{e}>u_{e}, u_{e}$ replaced by 0 whenever $e \notin A$, where $\psi_{e}=\sum_{i=1}^{k} \psi_{e}^{i}$ and $u_{e}=\sum_{i=1}^{k} u_{e}^{i}$.

(5) For each $e \in A$, if $e^{r}$ is reversed, $s_{c}\left(e^{r}\right)=u_{e}^{a}-\psi_{e}$ and $s_{c}(e)=0$. If neither $e$ nor $e^{r}$ is reversed, $s_{c}(e)=u_{e}-\psi_{e}>0$, where $s_{c}(e)$ is the saved capacity of $e$.

(6) Transform the solution to the original network.

Algorithm 1: MSMCF algorithm with partial lane reversals.

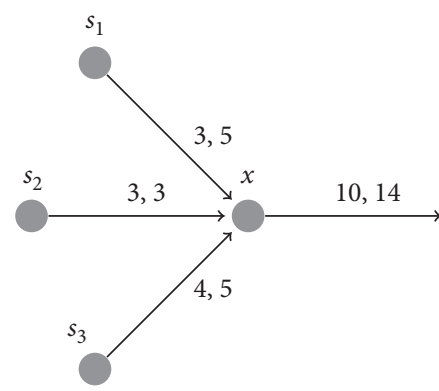

(a)

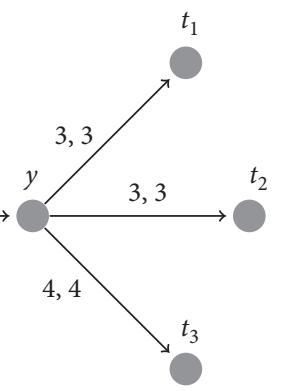

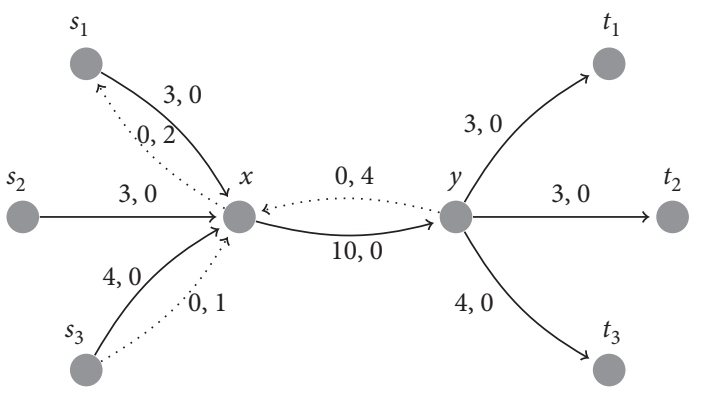

(b)

Figure 2: Network with (a) flow, capacity and (b) flow with saved capacity on the arcs.

TABle 1: Maximum multicommodity flow before and after lane reversals.

\begin{tabular}{lccc}
\hline Path & Flow before LR & Flow after LR & $\begin{array}{l}\text { \% of flow } \\
\text { increased }\end{array}$ \\
\hline$s_{1}-x-y-t_{1}$ & 2 & 3 & \\
$s_{2}-x-y-t_{2}$ & 2 & 3 & \\
$s_{3}-x-y-t_{3}$ & 0 & 4 & 150 \\
\hline Total & 4 & 10 & \\
\hline
\end{tabular}

$\mathrm{LR}=$ lane reversals

Proof. We know that the cut set $\mathscr{C} \subseteq A$ is the collection of disconnected and saturated arcs with the property that it disconnects sources and sinks. Let $\mathrm{Val}(\mathscr{C})$ be a value of cut which is the sum of the capacities of its individual arcs, i.e., $\operatorname{Val}(\mathscr{C})=\sum_{e \in \mathscr{C}} u_{e}$. We know that the arcs $e \in \mathscr{C}$ have twoway orientations with asymmetric (or symmetric) capacities. If each arc $e \in \mathscr{C}$ has the symmetric capacity, the lane reversal reconfiguration of the multicommodity network expands the capacity of cut two times. Let $\mathscr{C}^{a} \subseteq A^{a}$ be the cut set in the auxiliary network, then its value becomes

$$
\operatorname{Val}\left(\mathscr{C}^{a}\right)=\sum_{a \in \mathscr{C}^{a}} u_{e}^{a}=2 \sum_{e \in A} u_{e}
$$

The minimum multicommodity cut is

$$
\operatorname{Val}_{\min }\left(\mathscr{C}^{a}\right)=\min \sum_{a \in \mathscr{C}^{a}} u_{e}^{a} .
$$

Moreover, we have that every optimal maximum static multicommodity $\psi$ is less or equal to the minimum multicommodity cut in the auxiliary network. Consequently, the flow value can be increased up to double with lane reversals.

The reconfiguration of the multicommodity network with lane reversals is as follows:

$$
\operatorname{Val}_{\text {max }}(\psi)=\max \sum_{P^{a} \in \mathscr{P}^{a}} \psi\left(P^{a}\right) \leq \operatorname{Val}_{\text {min }}\left(\mathscr{C}^{a}\right)=2 \mathrm{Val}_{\text {max }}(\psi) \text {, }
$$

where $\mathscr{P}^{a}$ is the collection of all paths.

By Theorem 1, every optimal maximum static multicommodity flow in auxiliary network $\mathscr{N}^{a}$ is a feasible maximum static multicommodity flow with lane reversals in original network $\mathcal{N}$. This completes the proof.

3.2. Approximation Solution of MSMCF Problem. Linear programming techniques provide polynomial-time solutions for multicommodity flow problems. However, in many applications, these problems are large in input size and can take a long time to solve using these techniques. Due to this, it is beneficial to develop approximation algorithms that provide solutions close to the optimal solution. As a consequence, an intense attempt was made to obtain an efficient approximate algorithm for the multicommodity flow problem.

Let a minimization (or a maximization) problem be $\mathscr{X}$ and let the optimal solution of the objective function be denoted by $\operatorname{Opt}(I)$ for an instance $I \in \mathscr{X}$. Suppose $\varepsilon>0$. An algorithm $\mathscr{A}$ is called a $(1+\varepsilon)($ or $(1-\varepsilon))$ approximation 
algorithm for problem $\mathscr{X}$, if it produces a feasible solution with objective value $\mathscr{A}(I)$ for each instance $I$ of $\mathscr{X}$ such that

$$
\begin{aligned}
& |\mathscr{A}(I)-\operatorname{Opt}(I)| \leq \varepsilon \operatorname{Opt}(I) \\
& \quad \Rightarrow-\varepsilon \operatorname{Opt}(I) \leq \mathscr{A}(I)-\operatorname{Opt}(I) \leq \varepsilon \operatorname{Opt}(I), \\
& \Rightarrow(1-\varepsilon) \operatorname{Opt}(I) \leq \mathscr{A}(I) \leq(1+\varepsilon) \operatorname{Opt}(I)
\end{aligned}
$$

For a problem $\mathscr{X}$, a polynomial-time approximation scheme (PTAS) is an approximation scheme with the running time polynomial in the input size of the problem.

For a problem $\mathscr{X}$, a fully polynomial-time approximation scheme (FPTAS) is an approximation scheme with running time polynomial in the input size of the problem as well as polynomial in $1 / \epsilon$.

Fleischer [22] presented the first FPTAS for the maximum multicommodity flow problem that is independent of the number of commodities. It is faster than the best previous approximation schemes, whose running time is $O\left(\varepsilon^{-2} m^{2}\right)$. To calculate the approximate solution, Fleischer uses path flow linear programming formulation of a maximum multicommodity flow problem. The dual of this linear program corresponds to the problem of assigning length to the edges of the network such that the length of the shortest path from $s_{i}$ to $t_{i}$ is at least one for each commodity.

Let us assume that $l(e)$ and $l(P)$ denote the length of edges and length of the shortest path from $s_{i}$ to $t_{i}$ for each $i$, respectively. Algorithm starts with $l(e)=\delta, \forall e, \psi=0$, and selects a path $P \in \mathbb{P}$ with $l(P)<1$. The flow $\psi(P)=\psi(P)+u$ is assigned along the path $P$ where $u=\min _{e \in P} u(e)$, and the length $l(e)=l(e)(1+(\varepsilon u / u(e))), \forall e \in P$, of each edge is updated. The process is repeated until there exists a path with $l(P)<1$ otherwise return $(\psi, l)$.

Based on this, we present Algorithm 2 to calculate approximate solution of MSMCF with partial lane reversals.

Example 1 with capacity on arcs only (cf. Figure 2(a)) is considered, and maximum multicommodity flow with lane reversals is calculated.

Let us assume that $l(e)=0.25, \varepsilon=0.4$, and $\psi=0$. We choose the path $P_{1}: s_{1}-x-y-t_{1}$.

Since $l\left(P_{1}\right)=l\left(s_{1}, x\right)+l(x, y)+l\left(y, t_{1}\right)=0.25+0.25+$ $0.25=0.75<1$ and $u=\min _{e \in P_{1}} u_{e}=\min \{5,12,3\}=3$, we update the flow $\psi\left(P_{1}\right)=0+3=3$ and calculate the length of edges, i.e., $l\left(s_{1}, x\right)=l\left(s_{1}, x\right)\left(1+\left(\varepsilon u / u\left(s_{1}, x\right)\right)\right)=0.25(1+$ $((0.4 \times 3) / 5))=0.31$.

Similarly, $l(x, y)=0.275$ and $l\left(y, t_{1}\right)=0.35$. Therefore, $l\left(P_{1}\right)=0.31+0.275+0.35=0.935<1$. But, there is no path between $s_{1}$ and $t_{1}$ since edge $\left(y, t_{1}\right)$ is saturated.

We choose the next path $P_{3}: s_{3}-x-y-t_{3}$. Since $l\left(P_{3}\right)$ $=l\left(s_{3}, x\right)+l(x, y)+l\left(y, t_{3}\right)=0.25+0.25+0.25=0.75<1$ and $u=\min _{e \in P_{3}} u_{e}=\min \{5,9,4\}=4$, we update the flow value.

Now, $\quad l\left(s_{3}, x\right)=0.33, l(x, y)=0.283, l\left(y, t_{3}\right)=0.35$, and $l\left(p_{3}\right)=0.33+0.283+0.35=0.963<1$. But, there is no path between $s_{3}$ and $t_{3}$ since edge $\left(y, t_{3}\right)$ is saturated. Again, we update the flow value and length of the edges.
In the same way, the next path is $P_{2}: s_{2}-x-y-t_{2}$, $l\left(p_{2}\right)<1$ and $u=\min _{e \in P_{2}} u_{e}=3, \psi\left(P_{2}\right)=0+3=3$. There is no path from $s_{2}$ to $t_{2}$. Since there is no anymore $s_{i}-t_{i}$ path, the algorithm terminates, and maximum flow is $3+4+$ $3=10$.

Corollary 2. An approximate solution of MSMCF with partial lane reversals can be computed in fully polynomialtime complexity.

Proof. The complexity of the algorithm is dominated by Steps 2 and 3. All other steps can be computed in linear time. The solution to an approximate maximum multicommodity flow problem can be computed by Fleischer [22] on the auxiliary network in $O\left(\varepsilon^{-2} m^{2}\right)$ time in Step 2. This solution is equivalent to the maximum static multicommodity flow problem on the given network. The flow can be decomposed in $O(m n)$ time in Step 3. Hence, the MSMCF with partial lane reversals can be computed in fully polynomialtime.

\section{Dynamic Multicommodity Flow with Partial Lane Reversals}

In this section, we introduce the MDMCF with partial lane reversals by reverting the necessary arc capacities. Hall et al. [10] proved that multicommodity flow over time is $\mathscr{N} \mathscr{P}$-hard. They provided the solution of the flow over time problem in a network having uniform path length (a network where the length of all paths is the same).

The lane reversal strategy cannot be applied in case of uniform path length in general. It may violate the criterion of uniform path length after lane reversals as shown in example 3. Two algorithms to solve the MDMCF problem with lane reversals are presented in this section.

Example 3. Let us consider a multicommodity network where $s_{1}, s_{2}$, and $s_{3}$ are the source nodes and $t_{1}, t_{2}$, and $t_{3}$ are the sink nodes as shown in Figure 3(a). The first and second numbers on the arcs represent capacity and transit time associated with the arcs. The auxiliary network for reconfiguration is as shown in Figure 3(b). The network is of uniform path length before lane reversals and after the lane reversals, and a new path $s_{3}-x-y-t_{3}$ is created that violates uniform path length. In general, it proves that the lane reversal strategy fails to satisfy the condition of uniform path length. The lane reversal technique can be applied in case of uniform path length by reversing only those arcs that do not violate uniform path length.

4.1. Pseudopolynomial Solution of MDMCF. To deal with the maximum flow over time problem, Ford and Fulkerson [7] introduced the concept of time expansion. This well-known approach can be carried out in the case of the multicommodity flow over time problem. Kappmeier [11] has shown the equivalency between static multicommodity flow 
Input: given static multicommodity flow network $\mathcal{N}=\left(V, A, u, K, S_{+}, S_{-}\right)$

Output: the approximate MSMCF solution with partial lane reversals

(1) The given network is transformed by adding two-way capacities as $\mathscr{N}^{a}=\left(V, A^{a}, u_{e}^{a}, K, S_{+}, S_{-}\right)$.

(2) Compute the approximate MSMCF on the transformed network $\mathcal{N}^{a}$ by using the algorithm by Fleischer [22].

(3) Decompose the flow along the $s_{i}-t_{i}$ paths and cycles and remove flows in cycles $\forall i \in K$.

(4) Reverse $e^{r} \in A$ up to the capacity $\psi_{e}-u_{e}$ iff $\psi_{e}>u_{e}, u_{e}$ replaced by 0 whenever $e \notin A$, where $\psi_{e}=\sum_{i=1}^{k} \psi_{e}^{i}$ and $u_{e}=\sum_{i=1}^{k} u_{e}^{i}$.

(5) For each $e \in A$, if $e^{r}$ is reversed, $s_{c}\left(e^{r}\right)=u_{e}^{a}-\psi_{e}$ and $s_{c}(e)=0$. If neither $e$ nor $e^{r}$ is reversed, $s_{c}(e)=u_{e}-\psi_{e}>0$, where $s_{c}(e)$ is the saved capacity of $e$.

(6) Transform the solution to the original network.

Algorithm 2: An FPTAS for the MSMCF problem with partial lane reversals.

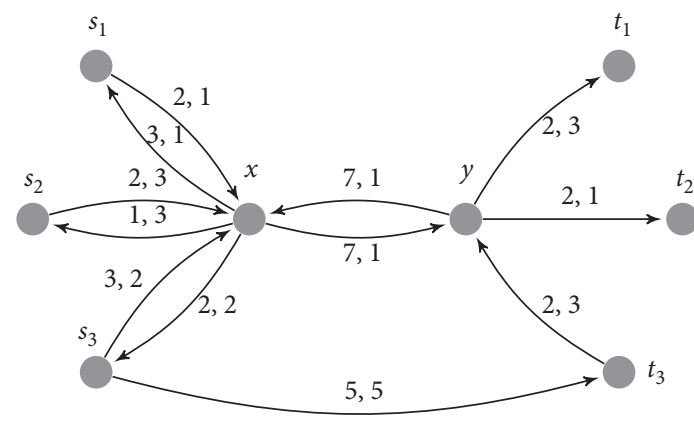

(a)

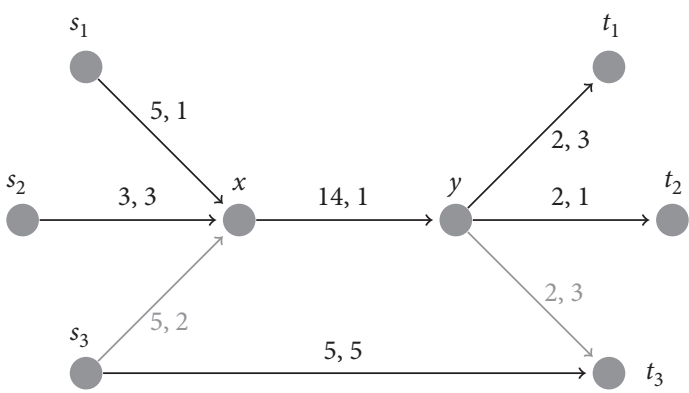

(b)

Figure 3: (a) Network with uniform path length before lane reversals (capacity, transit time). (b) Lane reversal network of (a) violating uniform path length (capacity, transit time).

on a time-expanded network and dynamic multicommodity flow on the original network as given below.

Lemma 1. Let $\mathcal{N}=\left(V, A, K, u, \tau, S_{+}, S_{-}, T\right)$ be a flow over time network. For any time horizon $T$, a feasible static $S_{+}^{\prime}-S_{-}^{\prime}$ multicommodity flow $\psi$ in the time-expanded network $\mathcal{N}_{T}$, there exists a feasible multicommodity flow over time $\xi$ with the sources $S_{+}$and sinks $S_{-}$in network $\mathcal{N}$ that sends the same amount of flow with time horizon $T$, i.e., $\left|\psi_{T}\right|=|\xi|$ and vice-versa.

Due to the reduction of the dynamic multicommodity flow problem on network $\mathcal{N}$ to the static multicommodity flow problem on a time-expanded network $\mathcal{N}_{T}$, it can be solved in pseudopolynomial-time complexity.

Problem 2. Consider a network $\mathcal{N}=\left(V, A, K, u, \tau, S_{+}\right.$, $\left.S_{-}, T\right)$. The MDMCF problem with partial lane reversals sends the maximum flow from the sources $s_{i}$ to the corresponding sinks $t_{i}$ in the unique pair of source and sink nodes $\left(s_{i}, t_{i}\right)$ for each commodity $i=1,2, \ldots, k$, and for a given time by saving the unused arc capacity.

To solve the maximum multicommodity flow over time problem with partial lane reversals (problem 2), we design Algorithm 3.

Theorem 3. Algorithm 3 provides pseudopolynomial-time solution of the MDMCF problem with partial lane reversals.
Proof. The theorem will be proved in three steps. In the first step, we show the feasibility. Step 2 of the algorithm is welldefined because it transforms the given dynamic network flow problem into the static network flow problem on the time-expanded auxiliary network. The feasibility of other steps in the algorithm can be shown as similar to Theorem 1. In the second step, we prove the optimality. Feasibility implies that any feasible solution of MDMCF on $\mathcal{N}_{T}^{a}$ is feasible to the MDMCF with lane reversal solution on network $\mathcal{N}$. Dynamic multicommodity flow problem on network $\mathcal{N}$ reduces to a static multicommodity flow problem on $\mathscr{N}_{T}^{a}$. By reducing the multicommodity to a single-commodity and decomposing the flow into the $\left(s_{i}-\right.$ $t_{i}$ ) path, dynamic multicommodity flow solution can be obtained optimally on auxiliary network $\mathscr{N}_{T}^{a}$. An optimal solution on $\mathscr{N}_{T}^{a}$ is equivalent to a feasible solution on $\mathscr{N}$. The unused capacities of the arcs are saved by partial lane reversals in Step 5. At last, we show that the algorithm solves it in pseudopolynomial-time. We know that Step 3 is solved in $O(m n)$ times, and Step 2 can be computed polynomially in the input size of the network, i.e., it depends on $T$. All other steps can be computed in linear time. Thus, Algorithm 3 solves the MDMCF problem with partial lane reversals on given network $\mathcal{N}$ optimally in pseudopolynomial-time complexity.

Example 4. We compute MDMCF on the auxiliary network obtained by adding two-way capacities of Figure 1(a) within 
Input: given dynamic multicommodity flow network $\mathcal{N}=\left(V, A, u, \tau, K, S_{+}, S_{-}, T\right)$

Output: the MDMCF with partial lane reversals

(1) A given dynamic network is transformed into time-expanded network by $\mathcal{N}_{T}=\left(V_{T}, A_{T}=A_{M} \cup A_{H} \cup\right.$

$\left.A_{+} \cup A_{-}, K, u, \tau, S_{+}^{\prime}, S_{-}^{\prime}, T\right)$

(2) An auxiliary network is constructed by adding two-way capacities as $\mathscr{N}_{T}^{a}=\left(V_{T}, A_{T}^{a}, u^{a}, \tau^{a} K, S_{+}^{\prime}, S_{-}^{\prime}, T\right)$.

(3) Compute the MSMCF on the auxiliary network $\mathcal{N}_{T}^{a}$.

(4) Decompose the flow along the $s_{i}-t_{i}$ paths and cycles and remove flows in cycles $\forall i \in K$.

(5) Reverse $e^{r}(\theta) \in A_{T}$ up to the capacity $\psi_{a}(\theta)-u_{e}$ iff $\psi_{a}(\theta)>u_{e}, u_{e}$ replaced by 0 whenever $e(\theta) \notin A_{T}$ where $\psi_{e}=\sum_{i=1}^{k} \psi_{e}^{i}$ and $u_{e}=\sum_{i=1}^{k} u_{e}^{i}$.

(6) For each $e(\theta) \in A_{T}$, if $e^{r}(\theta)$ is reversed, $s_{c}\left(e^{r}(\theta)\right)=u_{e}^{a}-\psi_{e}(\theta)$ and $s_{c}(e(\theta))=0$. If neither $e$ nor $e^{r}$ is reversed, $s_{c}(e(\theta))=u_{e}-\psi_{e}(\theta)>0$, where $s_{c}(e(\theta))$ is the saved capacity of $e$.

(7) Transform the solution to the original network.

Algorithm 3: Algorithm for MDMCF with partial lane reversals.

time horizon $T=8$. The repetition of path flows of each commodity is shown in a time-expanded network. We get static flow $\psi$ on the time-expanded network which corresponds to multicommodity flow over time $\xi$ on the auxiliary network. The maximum multicommodity flow over time computation after lane reversals is as shown in Figure 4.

4.2. Approximate Solution of MDMCF. A time-expanded network is a well-known technique to solve flow over time problems, but it has the drawback of a large blow-up of its size. By reducing the size of the time-expanded network, an efficient algorithm is presented. This reduction technique is known as condensation in the setting of a time-expanded network, and the network is known as the $\Delta$-condensed time-expanded network. If we take $\Delta=1$, then the $\Delta$-condensed time-expanded network reduces to the classical time-expanded network. To, solve Problem 2 in fully polynomial-time, we present Algorithm 4.

Theorem 4. Algorithm 4 provides approximate solution of the MDMCF problem with partial lane reversals.

Proof. First, we prove the feasibility. Step 1 of the algorithm is well-defined as it transforms the given dynamic network flow into the static network flow on the $\Delta$-condensed auxiliary network. The feasibility of other steps of the algorithm is similar to Theorem 1 .

Next, we prove the optimality. Feasibility implies that any approximate optimal solution of MDMCF with lane reversals on network $\mathcal{N}$ is also a feasible approximate solution to the MDMCF on $\mathcal{N}^{\Delta a}$. Dynamic multicommodity flow problem on network $\mathcal{N}$ reduces to a static multicommodity flow problem on $\mathscr{N}^{\Delta a}$. By reducing the multicommodity to a single commodity and decomposing it into the $\left(s_{i}-t_{i}\right)$ path, an approximate dynamic multicommodity flow solution can be obtained optimally on auxiliary network $\mathcal{N}^{\Delta a}$. An approximate optimal solution on $\mathcal{N}^{\Delta a}$ is a feasible solution on $\mathcal{N}$. The unused capacities of the arcs by partial lane reversals are saved in Step 5. Thus, an approximate MDMCF solution with lane reversals on each arc of the given network $\mathcal{N}$ can be computed optimally.
Corollary 3. An FPTAS to the MDMCF problem with partial lane reversal can be computed in fully polynomial-time complexity.

Proof. The complexity of Algorithm 4 is dominated by Steps 2 and 3. Step 3 is solved in $O(m n)$ time. An approximate solution of a static multicommodity flow problem on the $\Delta$-condensed auxiliary network is obtained by Fleischer [22] in $O\left(\varepsilon^{-2} m^{2}\right)$ time complexity in Step 2. The $\Delta$-condensed auxiliary network contains $\left(n^{2} / \varepsilon^{2}\right)$ nodes and $\left(m n / \varepsilon^{2}\right)$ arcs having polynomial-time complexity. Remaining steps can be solved in linear $O(m)$ time. So, the problem can be computed in fully polynomial-time.

By scaling the capacities and transit times on arcs given in Figure 1(b), $\Delta$-condensed networks are formed. The approximate solution of Problem 2 can be calculated by using the $\Delta$-condensed time-expanded network as shown in Figure 5 by taking $\Delta=2$.

The comparison of MDMCF before and after lane reversals is shown in Table 2 and Figure 6.

\section{EAMCT with Partial Lane Reversals}

The dynamic network topology $\mathcal{N}=\left(V, A, K, u, \tau, d_{i}\right.$, $\left.S_{+}, S_{-}, T\right)$ with set of commodities $K=\{1,2, \ldots, k\}$ is considered. The earliest arrival multicommodity flow problem is a feasible multicommodity flow over time $\xi$ with time horizon $T$ of a maximum value of $\xi(\theta)$ for each $\theta$. This problem becomes EAMCT if it is a feasible multicommodity transshipment over time $\xi$ with time horizon $T$ such that $\xi$ fulfills all supplies and demands $d_{i}$ and value of $\xi(\theta)$ is maximal at every point in time $\theta \in\{0,1, \ldots, T-1\}$.

Kappmeier [11] proved that the EAMCT exists in the case of multiple sources and a single-sink. They observed that it is possible to reduce the problem of the multicommodity to the single-commodity in the single sourcesink case. If all commodities commence in the same origin and have the same destination, then it can simply consider all commodities as one, compute the earliest arrival transshipment, and split the flow up into the commodities.

Problem 3. A network $\mathcal{N}=\left(V, A, K, u, \tau, d_{i}, S_{+}, t, T\right)$ with multisource and a single sink is considered. The EAMCT 


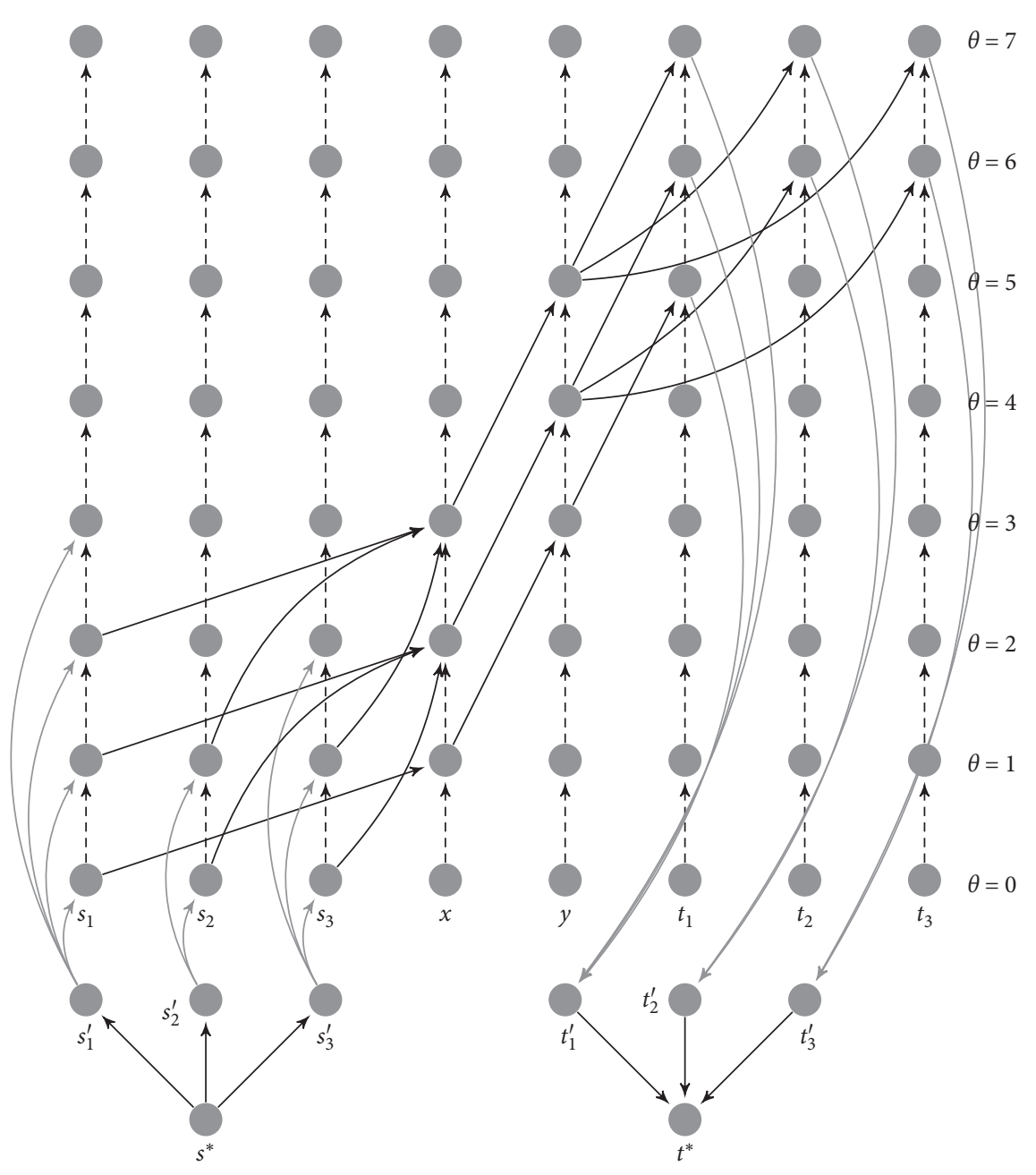

FIGURE 4: Time-expanded network of Figure 1(b).

Input: given dynamic multicommodity flow network $\mathcal{N}=\left(V, A, u, \tau, K, S_{+}, S_{-}, T\right)$

Output: the MDMCF with partial lane reversals

(1) The auxiliary network $\mathscr{N}^{a}$ is transformed to $\Delta$-condensed auxiliary network $\mathscr{N}_{T}^{\Delta a}=\left(V_{T}^{\Delta}, A_{T}^{\Delta a}, K, u^{\prime a}, \tau^{\prime a}, d_{i}^{\prime}, S_{+}^{\prime}, S_{-}^{\prime}, T\right)$ with $u_{e}^{\prime a}=\Delta\left(u_{e}+u_{e^{r}}\right), \tau_{e}^{\prime a}= \begin{cases}\left\lceil\tau_{e} / \Delta\right\rceil \Delta, & \text { if } e \in A, \\ \left\lceil\tau_{e^{r}} / \Delta\right\rceil \Delta, & \text { otherwise. }\end{cases}$

(2) Compute the approximate MSMCF by using algorithm by Fleischer [22] on the auxiliary network $\mathcal{N}_{T}^{\Delta a}$.

(3) Decompose the flow along the $s_{i}-t_{i}$ paths and cycles and remove flows in cycles $\forall i \in K$.

(4) Reverse $e^{r}(\theta) \in A_{T}^{\Delta}$ up to the capacity $\psi_{e}^{\prime}(\theta)-\Delta u_{e}$ iff $\psi_{e}^{\prime}(\theta)>\Delta u_{e}, u_{e}$ replaced by 0 whenever $e(\theta) \notin A_{T}^{\Delta}$ where $\psi_{e}^{\prime}=\sum_{i=1}^{k} \psi_{e}^{\prime i}$ and $u_{e}^{\prime}=\sum_{i=1}^{k} u_{e}^{\prime i}$.

(5) For each $e(\theta) \in A_{T}^{\Delta}$, if $e^{r}(\theta)$ is reversed, $s_{c}\left(e^{r}(\theta)\right)=u_{e}^{a}-\psi_{e}(\theta)$ and $s_{c}(e(\theta))=0$. If neither $e$ nor $e^{r}$ is reversed, $s_{c}(e(\theta))=u_{e}-\psi_{e}(\theta)>0$, where $s_{c}(e(\theta))$ is the saved capacity of $e$.

(6) Transform the solution to the original network.

Algorithm 4: An FPTAS algorithm for MDMCF with partial lane reversals.

problem with partial lane reversals sends the maximum flow from the sources $s_{i}$ to the corresponding sink $t$ in the unique source-sink pair $\left(s_{i}, t\right)$, for each commodity $i=1,2, \ldots, k$ and each time $\theta$ satisfying supplies and demands by saving the unused arc capacity.
Lemma 2. EAMCT with multiple sources and a single sink exists, and it can be computed with a running time that is polynomial in $k$ and size of the earliest arrival transshipment in the same network wherein all commodities are considered as one. 


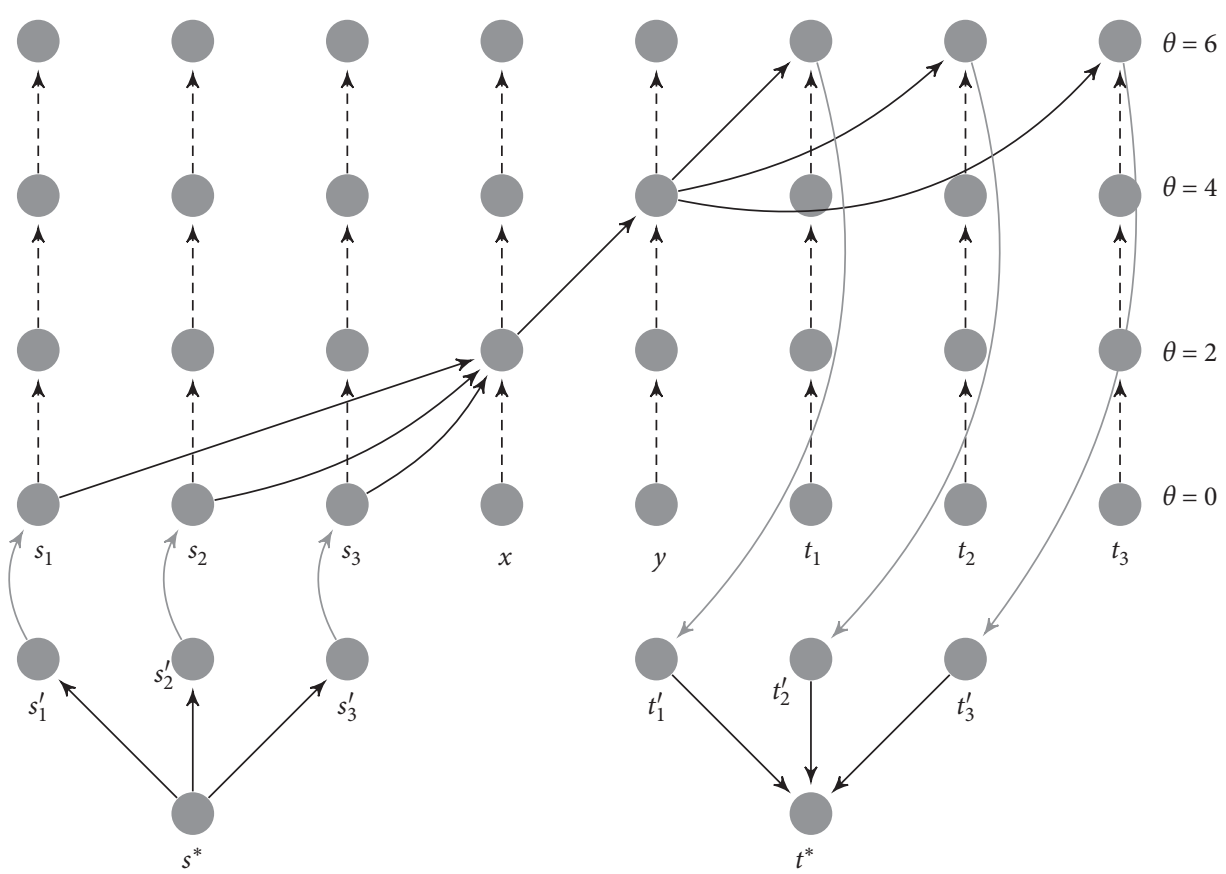

FIgURE 5: $\Delta$-condensed time-expanded network of Figure 1(b) after scaling capacity and transit time.

TABle 2: Maximum multicommodity flow over time with lane reversals.

\begin{tabular}{lcccc}
\hline & \multicolumn{2}{c}{ Time-expanded } & \multicolumn{2}{c}{$\Delta$-condensed } \\
Path & $\begin{array}{c}\text { Flow before } \\
\text { LR }\end{array}$ & $\begin{array}{c}\text { Flow after } \\
\text { LR }\end{array}$ & $\begin{array}{c}\text { Flow before } \\
\text { LR }\end{array}$ & $\begin{array}{c}\text { Flow after } \\
\text { LR }\end{array}$ \\
\hline$s_{1}-x-y-t_{1}$ & 6 & 9 & 4 & 6 \\
$s_{2}-x-y-t_{2}$ & 4 & 6 & 4 & 6 \\
$s_{3}-x-y-t_{3}$ & 0 & 8 & 0 & 8 \\
\hline Total & 10 & 23 & 8 & 20 \\
$\%$ increased & & 130 & & 150 \\
\hline
\end{tabular}

$\mathrm{LR}=$ lane reversals.

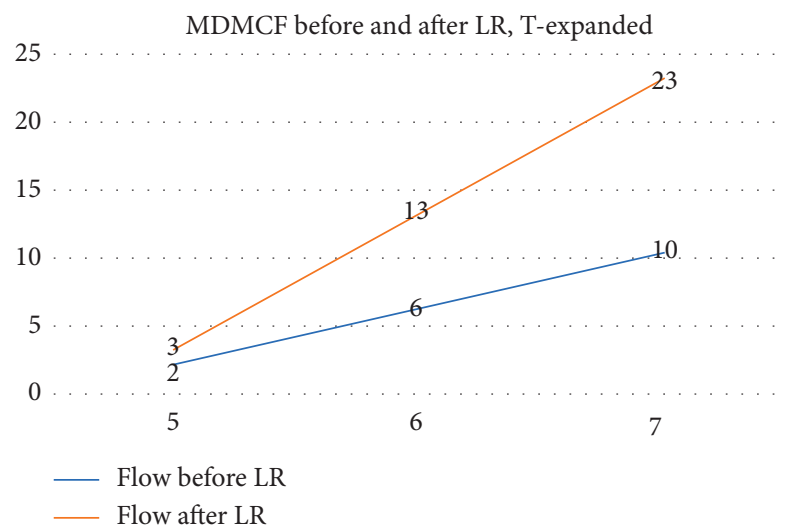

Figure 6: Comparison of flow before and after lane reversals.

Proof. Considering the flow over time network $\mathcal{N}=\left(V, A, K, u, \tau, d_{i}, S_{+}, t, T\right)$ having multiple sources $(k-$ sources) and a single-sink $t$ with $k$-commodities. Defining new supplies and demands $d^{\prime}$ by

$$
d_{v}^{\prime}= \begin{cases}\sum_{i \in K} d_{v}^{i}, & \text { if source } v \text { in } \cup_{i \in K} S_{+}^{i}, \\ \sum_{i \in K} d_{t}^{i}, & \text { if } v=t \text { is the sink } \\ 0, & \text { otherwise. }\end{cases}
$$

To compute an earliest arrival transshipment for the instance defined by the network $\mathcal{N}$, we first build the timeexpanded network with supplies and demands and calculate minimum cost flow by considering transit time as the cost using the successive shortest path algorithm. This yields multicommodity flow over time corresponding to the static multicommodity flow.

We split the path for each commodity $i \in K$ into commodity-dependent paths and select a source $s \in \cup_{i \in K} S_{+}^{i}$ with supply $d_{s}^{i}>0$. An $s-t$ path $P$ with a flow value $\xi_{P}>0$ is chosen, and $\xi_{P}^{i}=\min \left\{\xi_{p}, d_{s}^{i}\right\}$ is set as the flow value. Then, we update the residual demand $d_{s}^{i}=d_{s}^{i}-\xi_{P}^{i}$ and flow value $\xi_{P}=$ $\xi_{P}-\xi_{P}^{\prime i}$ and continue the process until there is no any source in $S_{+}^{i}$ with positive supply according to Kappmeier [11].

The above construction creates a feasible solution of the EAMCT problem. We have $\sum_{P \in \mathscr{P}_{\text {st }}} \xi_{P}=\sum_{i \in K} d_{v}^{i}$ by the definition of new demand for each source. $\xi_{P}^{\prime i}=\min \left\{\xi_{p}, d_{s}^{i}\right\}$ assures that no path capacity is violated as no more supply is sent exceeding the capacity. The flow value is reduced until arc capacities are zero, for any path $P$, and the constructed flow obeys the capacity constraint due to $\sum_{i \in K} \xi_{P}^{\prime i}=\xi_{P}$. Hence, feasible flow satisfying all demands is $\xi_{P}^{i}$ for all $i$. The flow obtained is the earliest arrival flow for a single-commodity setting. Thus, a path decomposition of the earliest arrival transshipment is computed in network $\mathcal{N}$. Selection of path flow for each 
Input: given a multicommodity flow network $\mathcal{N}=\left(V, A, K, u, \tau, d_{i}, S_{+}, t, T\right)$

Output: the EAMCT with partial lane reversals

(1) The given network is transformed to auxiliary network by adding two-way capacities in $\mathscr{N}^{a}=\left(V, A^{a}, K, u^{a}, \tau^{a}, d_{i}, \cup_{i \in K} S_{+}^{i}, t, T\right)$ as $u_{e}^{a}=u_{e}+u_{e^{r}}, \tau_{e}^{a}= \begin{cases}\tau_{e}, & \text { if } e \in A \\ \tau_{e^{r}}, & \text { otherwise. }\end{cases}$

(2) Compute the EAMCT on the transformed network $\mathscr{N}^{a}$ by using algorithm by Kappmeier [11].

(3) Decompose the flow along the $s_{i}-t$ paths and cycles and remove flows in cycles $\forall i \in K$.

(4) Reverse $e^{r}(\theta) \in A_{T}$ up to the capacity $\psi_{a}(\theta)-u_{e}$ iff $\psi_{a}(\theta)>u_{e}, u_{e}$ replaced by 0 whenever $e(\theta) \notin A_{T}$ where $\psi_{e}=\sum_{i=1}^{k} \psi_{e}^{i}$ and $u_{e}=\sum_{i=1}^{k} u_{e}^{i}$.

(5) For each $e(\theta) \in A_{T}$, if $e^{r}(\theta)$ is reversed, $s_{c}\left(e^{r}(\theta)\right)=u_{e}^{a}-\psi_{e}(\theta)$ and $s_{c}(e)=0$. If neither $e$ nor $e^{r}$ is reversed, $s_{c}(e(\theta))=u_{e}-\psi_{e}(\theta)>0$, where $s_{c}(e)$ is the saved capacity of $e$.

(6) Transform the solution to the original network.

Algorithm 5: EAMCT algorithm with partial lane reversals.
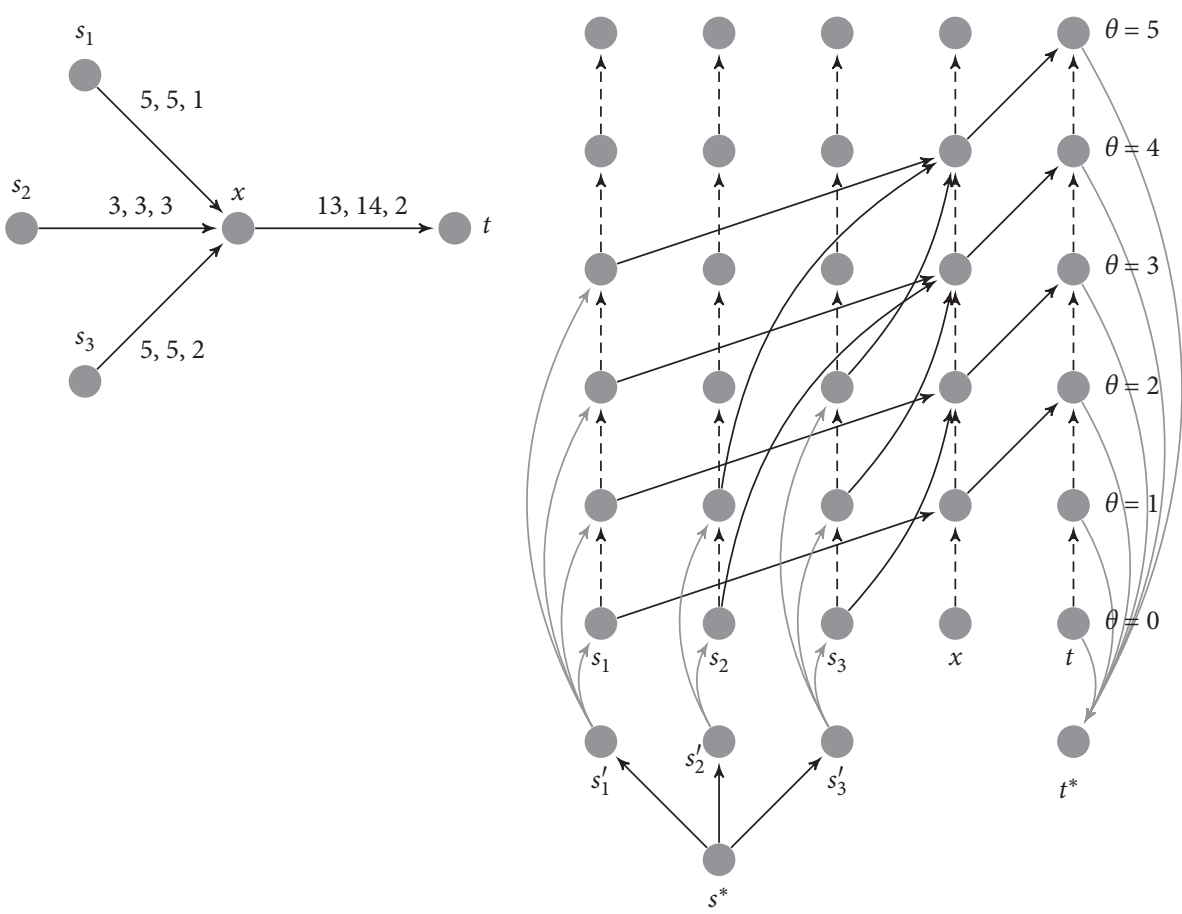

(a)

(b)

Figure 7: (a) Network having flow, capacity, and transit time on the arc after lane reversals. (b) Time-expanded network of (a).

commodity in polynomial-time in the size of the decomposition. Thus, running time is polynomial in $k$ and size of the input.

Earliest arrival multicommodity flow problem with partial lane reversals on network $\mathcal{N}=\left(V, A, K, u, \tau, d_{i}\right.$, $\left.S_{+}, t, T\right)$ is to find $S_{+}-t$ multicommodity flow over time that is maximum for all time steps $\theta \in\{0,1, \ldots T-1\}$ satisfying supply and demand of each commodity by reverting the arc partially. We present Algorithm 5 to calculate EAMCT with partial lane reversals.

Theorem 5. Algorithm 5 always computes an earliest arrival multicommodity flow with lane reversals on the multisource and single-sink network.
Proof. The theorem will be proved in two steps. In the first step, we show the feasibility of the algorithm, and in the second step, we show the optimality. Steps 1 and 4 of the algorithm are feasible according to Rebennack et al. [12]. The feasibility of Step 2 is verified by using the EAMCT algorithm (Kappmeier [11]). Step 3 of the algorithm is feasible being decomposition of flow along paths and cycles and assures that there is no cycle. The feasibility of Step 5 of the algorithm is due to Pyakurel et al. [19]. Thus, Algorithm 5 is feasible. To prove the optimality, we compute the EAMCT solution on the auxiliary network using the algorithm by Kappmeier [11]. From the feasibility of the algorithm, we conclude that every feasible EAMCT solution on the auxiliary network $\mathscr{N}^{a}$ is equivalent to the EAMCT solution with 
TABLE 3: EAMCT with partial lane reversals.

\begin{tabular}{lccc}
\hline Path & $\psi(P)$ & Repetition for $\theta$ & $\begin{array}{c}\text { Multicommodity } \\
\text { flow over time }\end{array}$ \\
\hline$s_{1}-x-t$ & 5 & $3,4,5,6$ & $\xi^{1}=20$ \\
$s_{3}-x-t$ & 5 & $4,5,6$ & $\xi^{3}=15$ \\
$s_{2}-x-t$ & 3 & 5,6 & $\xi^{2}=6$ \\
\hline Total & & & 41 \\
\hline
\end{tabular}

lane reversals in the original network $\mathscr{N}$. Thus, the solution obtained on the auxiliary network $\mathscr{N}^{a}$ is the EAMCT with lane reversals on the original network $\mathscr{N}$. Moreover, we can save the capacities of all unused arcs of the network by Step 5 of the algorithm.

Example 5. Considering a multisource and a single-sink network with demands for each commodity being $d_{1}=20, d_{2}=6$, and $d_{3}=15$, respectively. We compute maximum multicommodity flow over time on the auxiliary network as shown in Figure 7(a) with the flow, capacities, and transit times on the arcs within time horizon $T=6$ by searching the successive shortest path for each commodity on each time step $\theta$. The repetition of path flows of each commodity is shown in a time-expanded graph. We get static flow $\psi$ on the time-expanded graph which corresponds multicommodity flow over time $\xi$ on the auxiliary network. Computation of the EAMCT after lane reversals is shown in Figure 7(b).

Table 3 represents the value of EAMCT on each path with partial lane reversals.

\section{Conclusions}

One of the major problems in operational research is transshipping several kinds of commodities (goods) in underlying network topology, respecting capacity constraints on the arcs. Maximizing the sum of flow of all commodities in the specified period, it turns to maximum multicommodity flow over time. Multicommodity flow over time is computationally hard. A time-expanded network is a technique to solve flow over time problems, but it has pseudopolynomial-time complexity. By shrinking the size of the network, a $\Delta$-condensed time-expanded network is introduced without changing flow values too much, and it provided an efficient approximation scheme. By flipping the orientation of lanes, the capacity of the lanes will be increased that amplifies the flow value and reduces the time horizon. Partial lane reversal strategy reverses only necessary arc capacities and saves the capacity of unused arcs that can be used in case of emergency.

In this paper, we introduce a partial lane reversal strategy in static multicommodity flow, multicommodity flow over time, and earliest arrival multicommodity transshipment problems and provide algorithms to solve these problems. The first problem is solved in polynomial-time, the second problem is solved in pseudopolynomial-time as well as polynomial-time approximately, and the third problem is solved in pseudopolynomial-time complexity.

\section{Data Availability}

The authors have not used any additional data in this article.

\section{Conflicts of Interest}

The authors declare that there are no conflicts of interest regarding the publication of this paper.

\section{Acknowledgments}

The first author thanks the Alexander von Humboldt Foundation for the Return Fellowship (November 2019-October 2020). The second author thanks the University Grants Commission, Nepal, for the Ph.D. research fellowship.

\section{References}

[1] R. K. Ahuja, T. L. Magnanti, and J. B. Orlin, Network Flows, Theory Algorithms and Applications, Prentice-Hall, Englewood Cliffs, NJ, USA, 1993.

[2] A. A. Assad, "Multicommodity network flows-a survey," Networks, vol. 8, no. 1, pp. 37-91, 1978.

[3] J. L. Kennington, "A survey of linear cost multicommodity network flows," Operations Research, vol. 26, no. 2, pp. 209-236, 1978.

[4] K. Salimifard and S. Bigharaz, "The multicommodity network flow problem: state of the art classification, applications, and solution methods," Operational Research, pp. 1-47, 2020, In press.

[5] A. Ali, R. Helgason, J. Kennington, and H. Lall, "Technical note-computational comparison among three multicommodity network flow algorithms," Operations Research, vol. 28, no. 4, pp. 995-1000, 1980.

[6] J. A. Tomlin, "Minimum-cost multicommodity network flows," Operations Research, vol. 14, no. 1, pp. 45-51, 1966.

[7] L. R. Ford and D. R. Fulkerson, Flows in Networks, Princeton University Press, Princeton, NJ, USA, 1962.

[8] D. Gale, "Transient flows in networks," The Michigan Mathematical Journal, vol. 6, no. 1, pp. 59-63, 1959.

[9] N. Baumann and M. Skutella, "Earliest arrival flows with multiple sources," Mathematics of Operations Research, vol. 34, no. 2, pp. 499-512, 2009.

[10] A. Hall, S. Hippler, and M. Skutella, "Multicommodity flows over time: efficient algorithms and complexity," Theoretical Computer Science, vol. 379, no. 3, pp. 387-404, 2007.

[11] P. W. Kappmeier, "Generalizations of flows over time with application in evacuation optimization," Ph.D. thesis, Technical University, Berlin, Germany, 2015.

[12] S. Rebennack, A. Arulselvan, L. Elefteriadou, and P. M. Pardalos, "Complexity analysis for maximum flow problems with arc reversals," Journal of Combinatorial Optimization, vol. 19, no. 2, pp. 200-216, 2010.

[13] R. C. Dhungana and T. N. Dhamala, "Flow improvement in evacuation planning with budget constrained switching costs," International Journal of Mathematics and Mathematical Sciences, vol. 2020, Article ID 1605806, 10 pages, 2020.

[14] U. Pyakurel and T. N. Dhamala, "Models and algorithms on contraflow evacuation planning network problems," International Journal of Operations Research, vol. 12, pp. 36-46, 2015. 
[15] U. Pyakurel and T. N. Dhamala, "Continuous time dynamic contraflow models and algorithms," Advances in Operations Research, vol. 2016, Article ID 7902460, 7 pages, 2016.

[16] U. Pyakurel, T. N. Dhamala, and S. Dhamala, "Efficient continuous contraflow algorithms for evacuation planning problems," Annals of Operations Research, vol. 254, no. 1-2, pp. 335-364, 2017.

[17] H. N. Nath, U. Pyakurel, T. N. Dhamala, and S. Dempe, "Dynamic network flow location models and algorithms for quickest evacuation planning," Journal of Industrial and Management Optimization, 2020, In press.

[18] U. Pyakurel and T. N. Dhamala, "Evacuation planning by earliest arrival contraflow," Journal of Industrial and Management Optimization, vol. 13, no. 1, pp. 487-501, 2017.

[19] U. Pyakurel, H. N. Nath, S. Dempe, and T. N. Dhamala, "Efficient dynamic flow algorithms for evacuation planning problems with partial lane reversal," Mathematics, vol. 7, no. 10, pp. 1-29, 2019.

[20] T. N. Dhamala, S. P. Gupta, D. P. Khanal, and U. Pyakurel, "Quickest multi-commodity flow over time with partial lane reversals," Journal of Mathematics and Statistics, vol. 16, no. 1, pp. 198-211, 2020.

[21] L. Fleischer and M. Skutella, "Quickest flows over time," SIAM Journal on Computing, vol. 36, no. 6, pp. 1600-1630, 2007.

[22] L. K. Fleischer, "Approximating fractional multicommodity flow independent of the number of commodities," SIAM Journal on Discrete Mathematics, vol. 13, no. 4, pp. 505-520, 2000. 